\title{
Oligosaccharide Chromatographic Techniques for Quantitation of Structural Process-Related Impurities in Heparin Resulting From 2-O Desulfation
}

\author{
Pascal Anger ${ }^{1 *}$, Céline Martinez ${ }^{1}$, Pierre Mourier ${ }^{1}$ and Christian Viskov ${ }^{2}$ \\ ${ }^{1}$ Sanofi, Antony, France, ${ }^{2}$ Aspen NDB, Notre Dame de Bondeville, France
}

OPEN ACCESS

Edited by:

Elaine Gray,

National Institute for Biological Standards and Control (NIBSC),

United Kingdom

Reviewed by:

Edward Kelley Chess, BioPhia Consulting Inc., United States

Antonella Bisio,

Istituto di Ricerche Chimiche e

Biochimiche G. Ronzoni, Italy

${ }^{*}$ Correspondence:

Pascal Anger

pas-anger@orange.fr

Specialty section:

This article was submitted to

Hematology,

a section of the journa

Frontiers in Medicine

Received: 13 July 2018 Accepted: 23 November 2018 Published: 18 December 2018

Citation:

Anger $P$, Martinez $C$, Mourier $P$ and Viskov C (2018) Oligosaccharide Chromatographic Techniques for Quantitation of Structural

Process-Related Impurities in Heparin Resulting From 2-O Desulfation.

Front. Med. 5:346

doi: 10.3389/fmed.2018.00346
Heparin is a widely-used intravenous anticoagulant comprising a complex mixture of highly-sulfated linear polysaccharides of repeating sequences of uronic acids (either iduronic or glucuronic) 1->4 linked to D-glucosamine with specific sulfation patterns. Preparation of crude heparin from mammalian mucosa involves protease digestion with alcalase under basic conditions $(\mathrm{pH} \geq 9)$ and high temperature $\left(>50^{\circ} \mathrm{C}\right)$ and also oxidation. Under such conditions, side reactions including the ubiquitous 2-O desulfation occur on the heparin backbone yielding non-endogenous disaccharides within polysaccharide chains. Whatever the process used for its manufacture, some level of corresponding degradation impurities is therefore expected to be found in heparin and the derived Low Molecular Weight Heparins. These impurities should be monitored to control the quality of the final therapeutic product. Two anion exchange chromatography techniques were used to analyze heparin samples exhaustively or partially depolymerized with heparinases and determine the proportions of non-endogenous disaccharides generated by side reactions during the manufacturing process (epoxides and galacturonic moieties). We also present data from a case study of marketed heparin. Current heparin sodium monographs do not directly address process impurities related to modification of the structure of heparin. Although desulfation reduces the overall biological potency, we found that heparin with an average of one modified disaccharide per chain can still comply with the USP or Ph. Eur. heparin sodium monographs requirements. We have implemented disaccharide analysis to monitor the quality of this product on a risk basis.

Keywords: heparin, process, desulfation, impurities, pharmacopeias, chromatography

\section{INTRODUCTION}

Heparin is a key pharmaceutical product in the thrombosis field. It can be used therapeutically as unfractionated heparin (UFH), or as the starting material in the preparation of low-molecularweight heparins (LMWHs). UFHs are highly polydisperse mixtures of glycosaminoglycans (GAGs), with a typical average molecular weight of 15,000 Da. Its anticoagulant potency is mostly expressed in anti-factor IIa activity (anti-FIIa), which must be at least $180 \mathrm{U} / \mathrm{mg}$ according to the United States Pharmacopeia (USP) and European Pharmacopoeia (Ph. Eur.) monographs $(1,2)$. 
The purification processes from mucosa to pure heparin can usually be divided into three stages: (1) processing from mucosa to small batches of raw heparin; (2) consolidation of the small batches of raw heparin into crude heparin batches of variable size and; (3) purification from crude to pure heparin. The first stage often involves stabilization of mucosa with sodium metabisulfite, followed by alcalase depolymerization of heparin proteoglycan under basic conditions $(\mathrm{pH} \geq 9)$ and high temperature $\left(>50^{\circ} \mathrm{C}\right)$. Stage 3 of the heparin purification processes also involve basic conditions, with high temperatures $\left(>50^{\circ} \mathrm{C}\right)$ and different types of oxidation. Cationic resins or alkoxy ammonium precipitating agents and several steps of alcoholic precipitation are used in these process stages to isolate heparin from many impurities such as proteins, nucleic acids, and related GAGs (mainly dermatan sulfate and heparan sulfate).

The complexity of the supply chain in terms of geographical areas, number of raw heparin processing plants, and manufacturing processes raises potential concerns about the quality of heparin products at upstream stages due to the high number of contributors. Sanofi processes mucosa and crude heparin from different suppliers and therefore has a broad overview of the different manufacturing processes and consequently of the associated potential impurities.

The different USP and $\mathrm{Ph}$. Eur. monographs upgrades have dramatically improved the control of heparin quality but yet do not address directly process impurities derived from the heparin structure, i.e., the structural modification on the polysaccharide backbone. Instead the monograph specifications indirectly rely on increased activity requirement (anti-FIIa $\geq 180$ $\mathrm{U} / \mathrm{mg}$ for USP and Ph. Eur.) as a surrogate marker for structural integrity, which makes the accuracy and reproducibility of activity measurement crucial (3). As of July 2014, the Ph. Eur. has moved from requiring anticoagulant (anti-coag) to anti-FIIa activity measurements without modifying the specification of 180 $\mathrm{U} / \mathrm{mg}$. Although not always the case, it can be expected that most degradation reactions during the manufacturing process will alter antithrombin (AT) binding sites and ultimately induce some potency decrease. However, the acceptability of marginally degraded, although compliant, products may be questioned. Side reactions do not only occur around AT-binding sites and structural modifications that do not modify anti-FIIa activity may still have an impact on other biological properties of heparin (protein binding, $\mathrm{pK}$, etc.). Therefore, relying only on anti-FIIa or anti-coag activity may not be sufficient to capture the different modifications of the macromolecular backbone and ultimately control their potential biological effects.

Although different process structural impurities (4-7) impacting or not anti-IIa are known and either systematically present or present at sometimes significant levels, we here only focus on those resulting from the ubiquitous 2O-desulfation. We review this key side reaction and discuss how disaccharide and oligosaccharide analyses using strong anion-exchange (SAX) chromatography after heparinase digestions can be used to monitor these. We further present the findings of an investigation of these structural modifications undertaken as part of the follow-up on an out of specification (OOS) heparin batch. The discussed techniques can be used to further improve quality control at the raw/crude/pure stages of heparin production.

\section{MATERIALS AND METHODS}

\section{Reagents and Chemicals}

Sodium perchlorate $\left(\mathrm{NaClO}_{4}\right)$, monobasic sodium phosphate $\left(\mathrm{NaH}_{2} \mathrm{PO}_{4}\right)$, calcium acetate $\left(\mathrm{Ca}\left[\mathrm{C}_{2} \mathrm{H}_{3} \mathrm{O}_{2}\right]_{2}\right)$, and monobasic potassium phosphate $\left(\mathrm{KH}_{2} \mathrm{PO}_{4}\right)$ were purchased from VWR/Prolabo (Lutterworth, UK). Acetic acid $\left(\mathrm{C}_{2} \mathrm{H}_{4} \mathrm{O}_{2}\right)$ and bovine serum albumin (BSA) were obtained from Acros Organics (Geel, Belgium) and Sigma-Aldrich (St. Louis, MO, USA), respectively. Sodium hydroxide $(\mathrm{NaOH})$ and potassium hydroxide $(\mathrm{KOH})$ were purchased from Chemlab (Essex, UK). Phosphoric acid $\left(\mathrm{H}_{3} \mathrm{PO}_{4}\right)$ was obtained from Mallinckrodt (Phillipsburg, NJ, USA). All enzyme lyases from Flavobacterium heparinum (heparinase 1 [EC 4.2.2.7], heparinase 2 [no EC number], and heparinase 3 [EC 4.2.2.8]) were purchased from Grampian Enzymes (Aberdeen, United Kingdom). All other reagents and chemicals were of the highest quality available. Water was purified using a Millipore purification system (Guyancourt, France). Factor IIa and human AT were obtained from Stago (Asnières-sur-Seine, France). Factor Xa was of bovine origin and Factor IIa was of human origin. Enzymes and substrates [S2765 for Factor Xa and S2238 for Factor IIa] were obtained from Chromogenix (Paris, France). The USP heparin standard solution was used for anti-FXa and anti-FIIa activity measurements; Ph. Eur. standard solutions were used for anti-coag activity measurements. Ninety-six-well deep-well microplates for anti-FXa and anti-FIIa assays were obtained from Greiner Bio-One (Paris, France).

\section{Disaccharide Analysis Heparinase Digestions}

Exhaustive depolymerization of heparin was performed at room temperature for $48 \mathrm{~h}$ in a total volume of $190 \mu \mathrm{L}$ containing $20 \mu \mathrm{L}$ of $20 \mathrm{mg} / \mathrm{mL}$ heparin solution in water, $70 \mu \mathrm{L}$ acetate solution $\mathrm{pH} 7.0\left(2 \mathrm{mM} \mathrm{Ca}\left[\mathrm{C}_{2} \mathrm{H}_{3} \mathrm{O}_{2}\right]_{2}\right.$ and $\left.0.1 \mathrm{mg} / \mathrm{mL} \mathrm{BSA}\right)$, and $100 \mu \mathrm{L}$ heparinases $1+2+3$ solution containing $0.13 \mathrm{IU} / \mathrm{mL}$ of each heparinase in a $\mathrm{pH} 7.0$ potassium phosphate buffer $(10 \mathrm{mM}$ $\mathrm{KH}_{2} \mathrm{PO}_{4}$ and $0.2 \mathrm{mg} / \mathrm{mL}$ of BSA).

Partial digestion of heparin with heparinase 1 was performed at $16^{\circ} \mathrm{C}$ for $48 \mathrm{~h}$ with $10 \mathrm{mIU}$ heparinase 1 in a total volume of $130 \mu \mathrm{L}$ containing $20 \mu \mathrm{L}$ of $20 \mathrm{mg} / \mathrm{mL}$ heparin solution in water, $90 \mu \mathrm{L} 5 \mathrm{mM} \mathrm{NaH}_{2} \mathrm{PO}_{4}, \mathrm{pH} 7.0$, containing $200 \mathrm{mM} \mathrm{NaCl}$ and 2 $\mathrm{mg} / \mathrm{mL}$ BSA.

\section{Analysis of Heparin Digests With SAX Chromatography}

The comparative disaccharide analysis was performed using a gradient high-performance liquid chromatography (HPLC; Agilent 1100, Cheshire, UK) in SAX mode. Digested heparin solution $(8 \mu \mathrm{L})$ was injected onto a Spherisorb SAX 250 $\times 3.2 \mathrm{~mm}, 5 \mu \mathrm{m}$ column (Waters, Saint-Quentin en Yvelines, France). The column temperature was set at $50^{\circ} \mathrm{C}$, the flow rate to $0.45 \mathrm{~mL} / \mathrm{min}$, and $\mathrm{UV}$ detection was carried out at $234 \mathrm{~nm}$. Solvent A was $1.8 \mathrm{mM} \mathrm{NaH}_{2} \mathrm{PO}_{4}$ at $\mathrm{pH} 3.0$, and solvent $\mathrm{B}$ was 
an aqueous solution of $1.8 \mathrm{mM} \mathrm{NaH}_{2} \mathrm{PO}_{4}$ with $1 \mathrm{M} \mathrm{NaClO} 4$ adjusted to $\mathrm{pH}$ 3.0. A 50-min solvent $\mathrm{B}$ gradient with different slopes, starting from 3 to $100 \%$, was used and the highest solvent B\% flow was held for $10 \mathrm{~min}$. The integration was performed with Empower ${ }^{\circledR}$ (Waters, Saint-Quentin en Yvelines, France). Depolymerisation, HPLC, and quantification were performed as previously described (4). These procedures can also be found in the USP general chapter $<207>$ or $\mathrm{Ph}$. Eur. Enoxaparin monograph $(8,9)$.

\section{Analysis of Heparin Digests With Cetyltrimethylammonium (CTA)-SAX Chromatography}

The analysis of heparin digested by heparinase 1 was performed with cetyltrimethylammonium (CTA)-SAX chromatography as described previously (10). Briefly, $150 \times 2.1 \mathrm{~mm}$ columns filled with Kinetex $\mathrm{C}_{18} \quad 1.7 \mu \mathrm{m}$ particles (Phenomenex, Le Pecq, France) were dynamically coated using CTA using $1 \mathrm{mM}$ CTA $\mathrm{HSO}_{4}$ in $\mathrm{H}_{2} \mathrm{O}-\mathrm{CH}_{3} \mathrm{OH}(65: 35 \mathrm{v} / \mathrm{v})$ as the solvent for equilibration. Eluents used for CTA-SAX chromatography were as previously described (10). A linear concentration gradient (0-2 M) of aqueous ammonium methane sulfonate, $\mathrm{pH} 2.5$ was applied. Flow rate was set at $0.35 \mathrm{~mL} / \mathrm{min}$. Column temperature was $40^{\circ} \mathrm{C}$. Double UV detection was performed as described previously at $232 \mathrm{~nm}$ and at $202-242 \mathrm{~nm}$. The N-acetylated oligosaccharide selective signal, $202-242 \mathrm{~nm}$, is determined by subtracting the absorbance at $242 \mathrm{~nm}$ (reference signal) from that at $202 \mathrm{~nm}$ (detection wavelength).

\section{Analysis of Anti-coag, Anti-factor Ila, and Anti-factor Xa Activities}

Anti-coag activity, anti-factor IIa activity (anti-FIIa), and antifactor Xa activity (anti-FXa) were performed according to the USP general chapter $<208>$ and Ph. Eur. assay of heparin related to UFH $(11,12)$. Concentrations of standard and sample were assessed in duplicate. Three independent experiments were performed for each assay. All dilutions, dispensing, mixing, and control of incubation times were performed by the Microlab Star platform from Hamilton (Villebon sur Yvette, France). UV reading at end point was performed using EMax detector from Molecular Device (Sunnyvale, CA, USA). Statistical analysis was performed according to the Ph. Eur. $§ 5.3$ for parallel-line models (13). All activities were expressed on dry basis.

\section{Measurement of Mass Average Molecular Weight}

The mass average molecular weight (MW) was obtained by sizeexclusion chromatography (SEC). Each standard and sample (20 $\mu \mathrm{L}$ ) was injected onto two columns (TSKgel $5 \mu \mathrm{m}$ G3000SWXL and G2000SWXL $300 \times 7.8 \mathrm{~mm}$ ) used in series (TOSOH Biosciences, Lyon, France). Mobile phase was $0.5 \mathrm{M}$ lithium nitrate $\left(\mathrm{LiNO}_{3}\right)$, flow rate $0.6 \mathrm{~mL} / \mathrm{min}$, temperature $30^{\circ} \mathrm{C}$, and refractive index detection was used. Calibration was performed using a broad standard table associated with the internallyqualified standard, which is similar to the procedure described in the current USP Heparin Sodium monograph (1).

\section{RESULTS AND DISCUSSION}

\section{Degradation of Heparin in Basic Conditions}

The degradation mechanism of heparinoids in basic conditions was studied in depth to characterize the LMWH enoxaparin. In the enoxaparin purification process, modification of the heparin endogenous backbone occurs during depolymerization of the heparin ester when the heparin benzyl ester is heated in presence of $\mathrm{NaOH}$. Mechanistically, the cleavage of the heparin ester chain is the result of two competing chemical reactions, $\beta$ elimination and ester hydrolysis. Three key side reactions can occur after the $\beta$-elimination, caused by the alkalinity of the media: C-2 epimerization of the reducing end glucosamine to mannosamine in alkaline media $(14,15)$; 6-O desulfation with the formation of a 1,6-anhydro ring at the reducing end; and 2-O desulfation of uronic acids. The first two side reactions observed during enoxaparin purification, while theoretically possible, are normally marginal for heparin due to the low amount of reducing ends available. However, 2-O desulfation of uronic acids has been reported as one of the main mechanisms of heparin degradation $(16,17)$. Although minor amounts of 2-O sulfated glucuronic acids have been previously reported in heparin (18), the vast majority of 2-O sulfated uronic acids have an iduronic configuration. An epoxy intermediate compound also obtained during 2-O desulfation further hydrolyses into either an iduronic acid or a galacturonic acid (Figure 1).

Desulfation can occur at all disaccharides with a 2-O-sulfated uronic acid moiety, i.e., Is, IIIs, Ia, and IIIa. However, as their typical weight percentages $(\mathrm{w} / \mathrm{w} \%)$ in heparin are $60,6,1$, and $1 \%$ respectively, only $\mathrm{IIs}_{\text {gal }}$ and $\mathrm{IVs}_{\text {gal }}$ are readily identifiable by chromatographic analysis. Due to its abundance, we will focus more on IIs saccharides derived from Is, which comprise the natural disaccharide IIs and the two non-endogenous disaccharides IIs $s_{\text {gal }}$ and IIs $s_{\text {epoxy }}$ (Figure 1). Nevertheless, we will also give some information on $\mathrm{IVs}_{\mathrm{gal}}$ formation.

\section{Quantitation of the Overall 2-O Desulfation Phenomenon}

Analytical quantitation of the overall 2-O desulfation is not straightforward.

Chromatographic analysis of the disaccharides generated after exhaustive depolymerisation by heparinases mixture allows the monitoring of two main markers of 2-O desulfation i.e., $\Delta I I s_{\text {gal }}$ and $\Delta I V s_{\text {gal }}$ (Figure 2). This analysis is straightforward and should be implemented first when investigating potential degradation of heparin under basic conditions. An appropriate method is available $(4,8,9)$. IIs is also generated, and adds to the already significant endogenous IIs content in heparin $(\sim 10 \%)$ (Figure 1).

Epoxide disaccharides cannot be assessed after exhaustive depolymerisation by heparinase mixture as this non-endogenous derivative is poorly recognized by heparinases and the disaccharide bearing the epoxy is very unstable. It is actually rapidly degraded after a probable mono-desulfation.

In a partially 2 -O desulfated heparin, the -GlcNS6S $\downarrow$ IIs epoxy site cannot be cleaved by heparinase 1 . When heparinase 2 is 


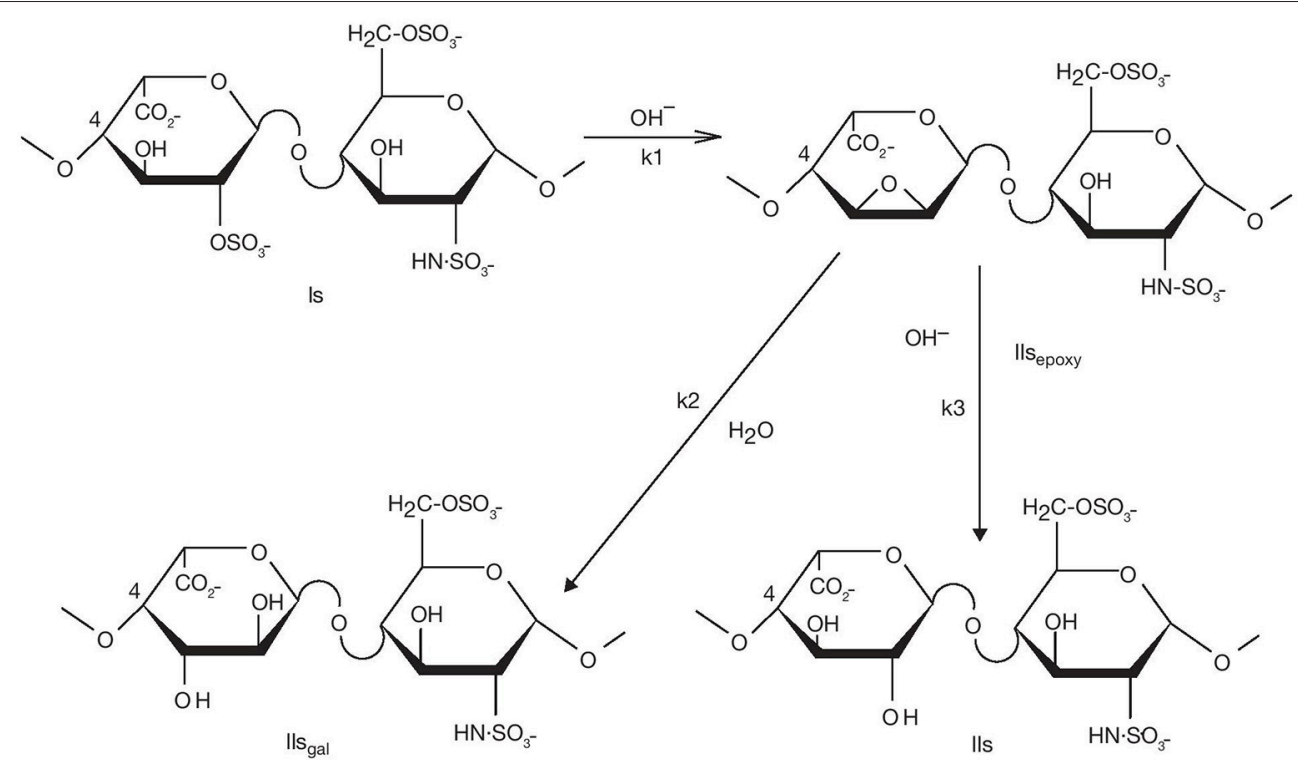

FIGURE 1 | Desulfation of the Is disaccharide in basic conditions.

used, the cleavage becomes possible, but has a slow reaction kinetic. Heparinase 3 has no action.

Bovine lung heparin is more homogeneous and more 2-O sulfated than porcine intestinal mucosa heparin and thus easier to study. In previous experiments, incomplete depolymerisation was obtained when partially 2-O desulfated (30 and $60 \mathrm{~min}$ in $1 \mathrm{~N} \mathrm{NaOH}, 60^{\circ} \mathrm{C}$ ) bovine lung heparin was exposed to the heparinase mixture. Although tetrasaccharides $\Delta \mathrm{IIs}_{\mathrm{gal}^{-}}$ IIs epoxy $_{\text {and }} \Delta \mathrm{IIs}_{\text {epoxy }}$-IIs epoxy $_{\text {ere identified (unpublished }}$ results), the disaccharide $\Delta \mathrm{IIs}_{\text {epoxy }}$ was not detected. If the same 2-O desulfated heparin is treated by heparinase 1 alone, consecutive epoxidized disaccharides $\left(\mathrm{IIs}_{\text {epoxy }}\right)_{\mathrm{n}}$ sequences are resistant to depolymerization. Gel permeation chromatography (GPC) subsequently reveals a mixture of oligosaccharides ranging from disaccharides to oligosaccharides larger than dodecasaccharides (in comparison, when typical porcine heparin is depolymerized with heparinase 1, the largest observable hexasaccharide is the truncated AT binding site $\left.\Delta \mathrm{Is}^{-\mathrm{IIa}_{\mathrm{id}}}-\underline{\mathrm{II}}_{\mathrm{glu}}\right)$.

The main tetrasaccharides generated during heparinase 1 digestion, $\Delta \mathrm{Is}_{\mathrm{III}} \mathrm{s}_{\mathrm{gal}}$ and $\Delta \mathrm{Is}$-IIs $_{\text {epoxy, }}$ have been purified and identified (10). The structure of the most predominant

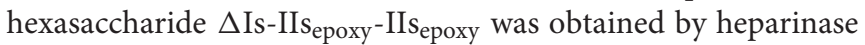
sequencing. When $\Delta$ Is-IIs $_{\text {epoxy }}$ is exposed to heparinase 2, only $\Delta$ Is is obtained. When $\Delta$ Is-IIs epoxy $-\mathrm{IIs}_{\text {epoxy }}$ is treated by heparinase 2 , only a mixture of $\Delta \mathrm{Is}, \Delta \mathrm{Is}^{-\mathrm{II}_{\text {epoxy }}}$ and $\Delta \mathrm{IIs}_{\text {epoxy }}{ }^{-}$ IIs $s_{\text {epoxy }}$ is obtained. These data suggest that the $\Delta \mathrm{IIs}_{\text {epoxy }}$ disaccharide may be either unstable or degraded by heparinase 2 , and thereby cannot be quantified directly.

2D-NMR can be used to quantitate epoxidation in heparinoid products (19) and has been successfully applied to crude heparins (20). However, this analytical method cannot be considered a routine technique for a Quality Control laboratory.
As both 2D-NMR and disaccharide analysis after exhaustive depolymerisation have limitations to assess the overall 2-O desulfation pattern in crude heparin, we have developed the following additional chromatographic analysis (10).

\section{Quantitation of Ilsepoxy After Partial Depolymerisation Analysis of Heparinase 1 Digests With CTA-SAX Chromatography}

As indicated previously, the major components of the mixture obtained after heparinase 1 digestion of porcine mucosa heparin have already been determined. However, the complexity of the mixture obtained makes a quantitative assay of all the components very challenging. In this quantitation method, specifically developed for porcine mucosa heparin, sufficient amounts of heparinase 1 is added to bring the reaction to completion. The resulting digest is a heterogeneous mixture of disaccharides, tetrasaccharides, and hexasaccharides (Figure 3).

Two chromatograms corresponding to heparinase 1 digests of a typical crude heparin (100101) and a partially desulfated crude heparin (9302) differing by their level of 2-O desulfation are shown in Figures 3A,B, respectively. The additional peaks in the chromatogram corresponding to the partially desulfated heparin (Figure 3B) are highlighted with arrows and can be attributed to saccharidic moieties transformed by $\mathrm{NaOH}$, in addition to the well-characterized (desulfated) $\Delta$ Is-IIs $_{\text {epoxy }}$ and $\Delta I s-I I s_{\text {gal }}$ moieties.

Although $\Delta$ Is-IIs $_{\text {epoxy }}$ and $\Delta$ Is-IIs $_{\text {gal }}$ are the most important oligosaccharides containing IIs epoxy $_{\text {and IIs }}$, just using the absolute levels of these identified molecules would significantly underestimate the overall levels of the desulfated saccharides and therefore overall degradation. Nevertheless, we can select $\Delta$ Is-IIs $_{\text {epoxy }}$ and $\Delta$ Is-IIs $_{\text {gal }}$ as good potential markers to estimate 


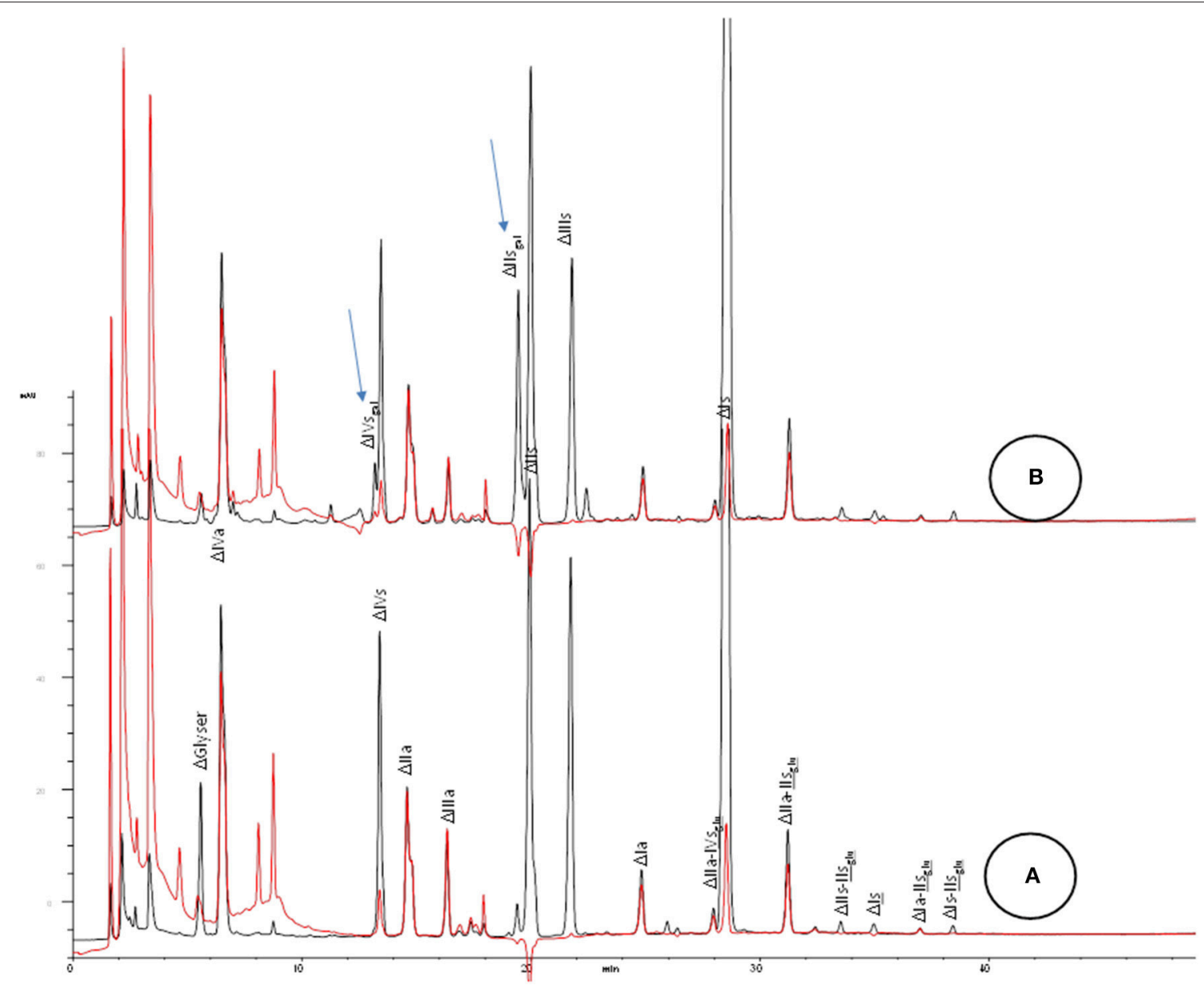

FIGURE 2 I SAX analysis of crude heparin batches after heparinase 1+2+3 depolymerisation for (A) typical heparin; (B) partially 2-O desulfated heparin. Black line: UV 234 nm; red line: UV 202 nm-242 nm. Arrows for assumed desulfated oligosaccharides.

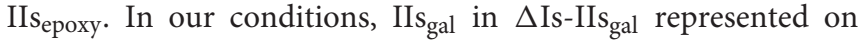
average more than $40 \%$ of the overall IIs gal $_{\text {al }}$ as obtained by depolymerization of heparin with heparinase $1+2+3$, thereby confirming the value of this marker for use in quantitation.

\section{Calculation of IIs epoxy}

We here evaluate the entire content of IIs $s_{\text {epoxy }}$ using:

- the 2 previously mentioned main markers $\Delta$ Is-IIs epoxy $_{\text {and }}$ $\Delta$ Is-IIs $_{\text {gal }}$ easily obtained after heparinase 1 digestion

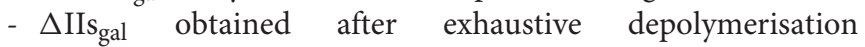
(heparinases $1+2+3$ ); this analysis is well-established as quantitation for the entire content of IIs $s_{\text {gal }}$ (or IIs $s_{\text {gal }}$ )

We then make the assumption that the ratio $\mathrm{k}$ of the entire content of IIs epoxy (from the different desulfated species seen Figure $3 B$ ) to IIs epoxy in $\Delta$ Is-IIs epoxy is the same as that of the entire content of IIs $_{\text {gal }}$ (from the different desulfated species seen Figure 3B) to IIs gal in $\Delta \mathrm{Is}_{\mathrm{II}} \mathrm{II}_{\text {gal }}$. The validity of this assumption is limited by the differences of depolymerisation

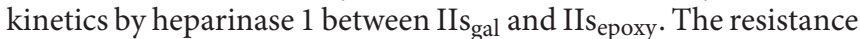

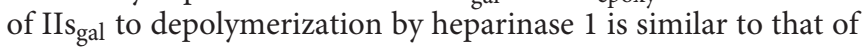

IIs glu $_{\text {, so that }} 5-10 \%$ of their total amount are already present

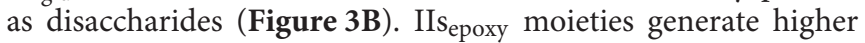
resistance to depolymerization, so that the mean molecular

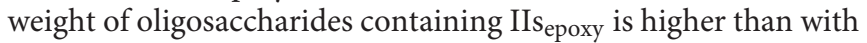
IIs $_{\text {gal }}$ which could led to different proportions of $\Delta$ Is-IIs epoxy compared to $\Delta$ Is-IIs $_{\text {gal }}$. From heparinase 1 digestion we have:

entire content of IIs epoxy $\left(\right.$ or IIs $\left.s_{\text {epoxy }}\right)=k \times I I s_{\text {epoxy }}$ in $\Delta I s-I I s_{\text {epoxy }}$ and entire content of IIs $s_{\text {gal }}\left(\right.$ or IIs $\left.s_{\text {gal }}\right)=k \times I I s_{\text {gal }}$ in $\Delta I s-I I s_{\text {gal }}$ (1)

As IIs $s_{\text {epoxy }}$ and IIs $s_{\text {gal }}$ have similar molecular weights, we can write the mass contents of IIs $s_{\text {epoxy }}$ in $\Delta$ Is-IIs $s_{\text {epoxy }}$ and IIs $s_{\text {gal }}$ in $\Delta$ Is-IIs $s_{\text {gal }}$

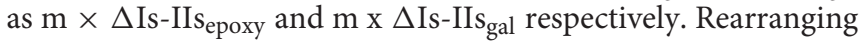
(1), we have:

$$
k=\frac{I I s_{\text {epoxy }}}{I I s_{\text {epoxy }} \text { in } \Delta I s-I I s_{\text {epoxy }}}=\frac{I I s_{\text {gal }}}{I I s_{\text {gal }} \text { in } \Delta I s-I I s_{\text {gal }}}
$$

and then

$$
k=\frac{I I s_{\text {epoxy }}}{m \times \Delta I s-I I s_{\text {epoxy }}}=\frac{I I s_{\text {gal }}}{m \times \Delta I s-I I s_{\text {gal }}}
$$




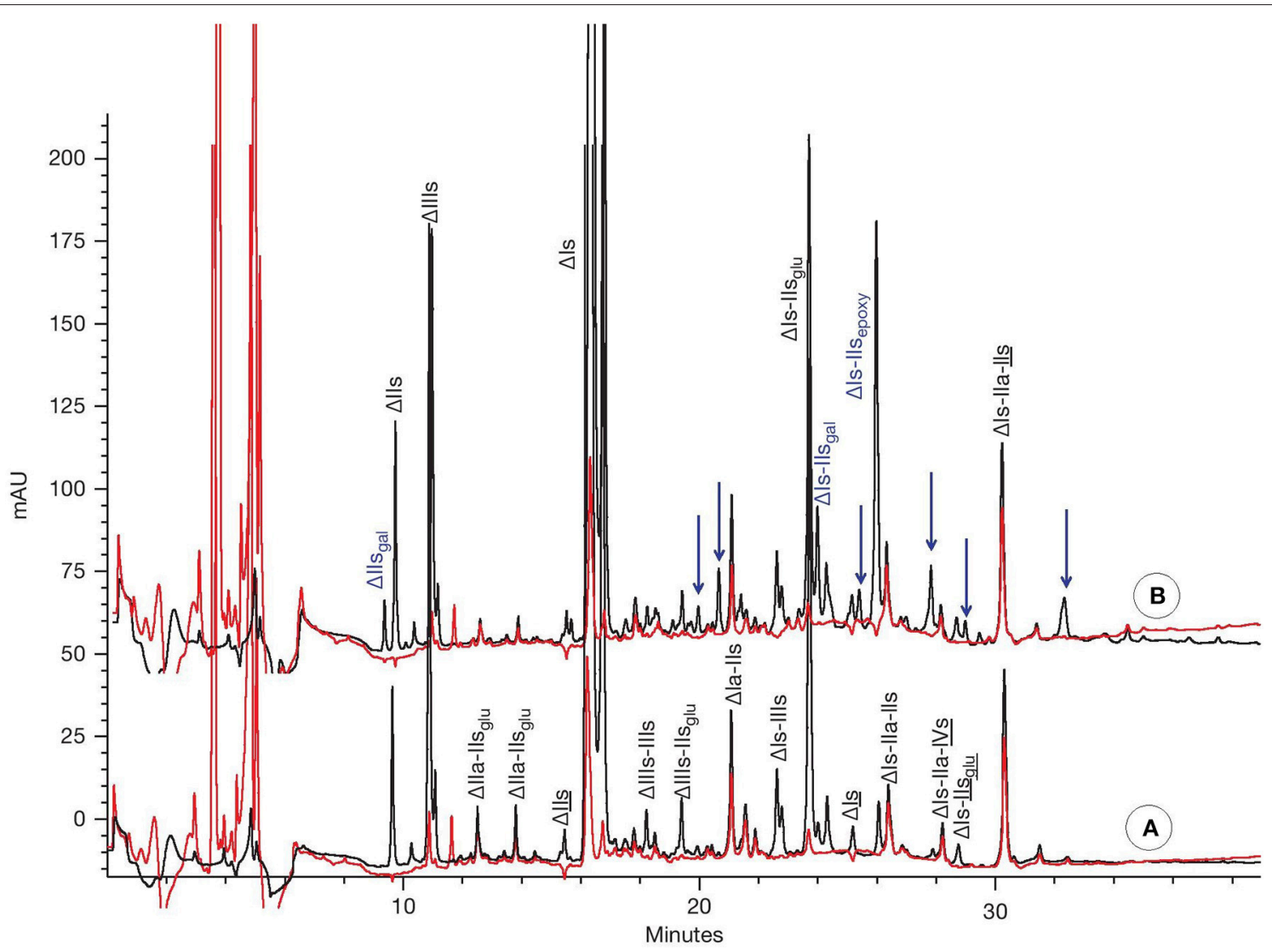

FIGURE 3 | CTA-SAX analysis of heparin batches after heparinase 1 depolymerisation for (A) typical heparin; (B) partially 2-O desulfated heparin. Black line: UV $234 \mathrm{~nm}$; red line: UV 202-242 nm. Arrows for assumed desulfated oligosaccharides.

or

$$
\frac{I I s_{\text {epoxy }}}{\Delta I s-I I s_{\text {epoxy }}}=\frac{I I s_{\text {gal }}}{\Delta I s-I I s_{\text {gal }}}
$$

As indicated above, IIs gal $_{\text {is }}$ obtained from (heparinases $1+2$ +3 ) digestion whereas $\Delta$ Is-IIs $_{\text {epoxy }}$ and $\Delta$ Is-IIs $_{\text {gal }}$ are obtained from heparinase 1 digestion.

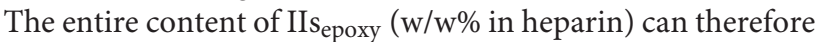
be estimated as follows:

$$
\begin{array}{r}
I I s_{\text {epoxy }} w / w \%=\left(\Delta I I s_{\text {gal }} \text { by heparinases } 1+2+3\right) w / w \% \\
\quad \times\left(\frac{\Delta I s I I s_{\text {epoxy }}}{\Delta I s I I s_{\text {gal }}} \text { by heparinase } 1\right) .
\end{array}
$$

\section{Other Parameters Impacted by Heparin Degradation Under Basic Conditions}

The degradation of heparin impacts the activity (anti-coag, anti$\mathrm{IIa}$, anti-Xa) and the MW of heparin. It has been shown that 2-O desulfation of the Is disaccharide from the AT pentasaccharide binding sequence $\mathrm{IIa}_{\mathrm{id}} \mathrm{IIs}_{\mathrm{glu}} \mathrm{I} s_{\mathrm{id}}$ results in a complete loss of activity (21). The different activities (anti-FIIa, anti-FXa, and anti-coag) were measured using the methods currently described in the USP general chapter 208 and Ph. Eur. assay of heparin $(11,12)$. MW characterization was performed with a method similar to that described in the USP heparin sodium monograph (1) - except for the standard, which was not available at the time of study.

\section{Supplier Case Study Background}

Our company purchases crude heparin material from several suppliers to manufacture pure heparin, which is then tested for compliance with current USP and Ph. Eur. specifications. The purification of heparin is performed using batches from a single crude heparin supplier so that pure heparin batches remain traceable to a single supplier. Pure heparin batch 5720 manufactured from crude heparin from supplier A was found to be compliant with the Ph. Eur. heparin sodium monograph for all tests except anti-coag activity (177 U/mg; out of specification of $\geq 180 \mathrm{U} / \mathrm{mg}$ ); anti-coag activity was the compendial test at the time of the finding. Measurement of IIs gal and IVs gal led to an estimate of at least $7 \% 2-\mathrm{O}$ desulfation $\left(\Delta \mathrm{II} s_{\text {gal }}+\Delta \mathrm{IV} \mathrm{s}_{\text {gal }}\right)$ in this sample (Table 1). To investigate further whether the loss of activity could be linked to the magnitude of $2-\mathrm{O}$ desulfation reactions, we applied our previously described analytical methodology to other heparin batches. Due to this OOS issue, 


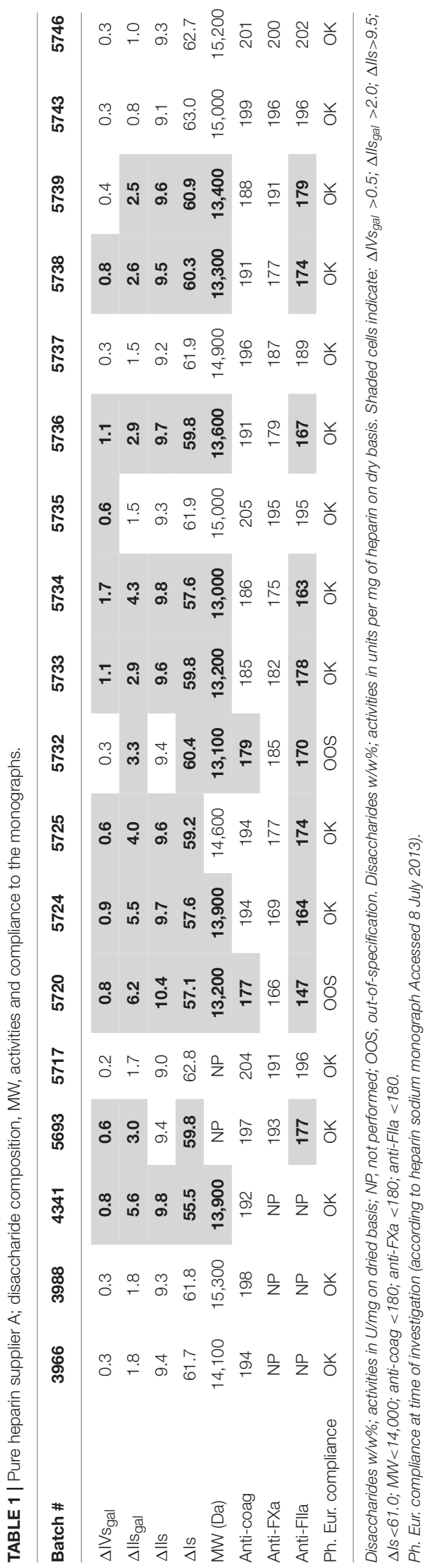

we undertook an in-depth investigation on the batches from supplier A.

Conclusions drawn here result from data reported in this paper and additional historical data.

\section{Disaccharide Analysis After Exhaustive Depolymerization}

$\Delta \mathrm{IIs}_{\text {gal }}$ contents of crude heparin batches from our different suppliers are periodically evaluated and used to build a database. Suppliers C and D have levels always lower than $1.0 \%$ whereas suppliers A and B have higher levels, with an average of $\sim 2 \%$. For pure heparin, the maximum $\Delta \mathrm{IIs}_{\text {gal }}$ content found was $6.2 \%$ for batch 5720 (as previously indicated) from supplier A (Table 2). In contrast, the maximum $\Delta \mathrm{IIs}_{\mathrm{gal}}$ content found in crude heparin used to manufacture this batch was only $2.4 \%$. Interestingly, although suppliers A and B have similar levels for crude heparin batches, pure heparin batches from supplier $\mathrm{B}$ had a maximum $\Delta \mathrm{IIs}_{\text {gal }}$ content $2.0 \%$ compared with $\sim 6 \%$ for supplier A. This shows that IIs gal content can remain stable, or substantially increase during the purification of crude into pure heparin depending of the batches used, even when the same purification process is used. Disaccharide analysis of crude heparin is therefore not sufficient to predict the quality of the expected pure heparin.

\section{Evaluation of Total Degradation}

IIs $_{\text {epoxy }}$ content was obtained using a combination of both analyses (heparinases $1+2+3$ and heparinase 1 ) as previously described. All results are reported (Table 2). Total degradation was evaluated by adding IIs epoxy to $\Delta$ IIs $_{\text {gal }}$.

\section{Respective Importance of $\Delta \mathrm{Ils}_{\text {gal }}$ and IIs $\mathrm{s}_{\mathrm{ex}}$ as 2-O Desulfation Markers in Pure and Crude Heparin Batches}

In pure heparin obtained from crude from suppliers $\mathrm{A}, \mathrm{B}, \mathrm{C}$, and D, IIs $s_{\text {epoxy }}$ was approximately half $\Delta \mathrm{IIs}_{\text {gal }}$ content $\left(\frac{\mathrm{II} I_{\text {epoxy }}}{\Delta I I_{\text {gal }}}\right.$ between 33 and $83 \%$ [mean 58\%]), whereas in crude heparin it varied from close to 0 for supplier B up to between 125 and $327 \%$ (mean 260\%) for supplier A; thus making IIs epoxy by far the most important 2-O desulfation marker in crude heparin from supplier A. In crude heparin, $\Delta \mathrm{II} s_{\text {gal }}$ therefore represented on average $100 \%$ of the total desulfation for supplier B, down to $28 \%$ for supplier $\mathrm{A}$. As a consequence, $\Delta \mathrm{II}_{\text {gal }}$ alone cannot be used a priori as a reliable marker to estimate the magnitude of crude heparin desulfation. In pure heparin samples, $\Delta$ IIs $_{\text {gal }}$ was found to be a more reliable indicator due to the partial hydrolysis of IIs epoxy $_{\text {into }}$ IIs $_{\text {gal }}$ during the purification process used, thereby decreasing the bias observed when not taking into account IIs epoxy species. The heparin purification process used hydrolyses most, but not all, IIs epoxy $_{\text {to }}$ IIs gal $_{\text {. }}$.

The total amount of $\left(\mathrm{IIs}_{\text {epoxy }}+\mathrm{IIs}_{\mathrm{gal}}\right) \%$ in crude batches was found similar to that in the derived pure heparin batches (Table 2). In addition to the absence of IIs gal increase (supplier $\mathrm{B}, \mathrm{C}$, and $\mathrm{D}$ ), this suggests that the manufacturing purification process used does not further 2-O desulfate heparin and that the

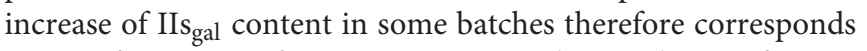
to transformation of IIs $s_{\text {epoxy }}$ into IIs $s_{\text {gal }}$ during the purification 
TABLE 2 | Disaccharide analysis of pure heparin and crude heparin batches.

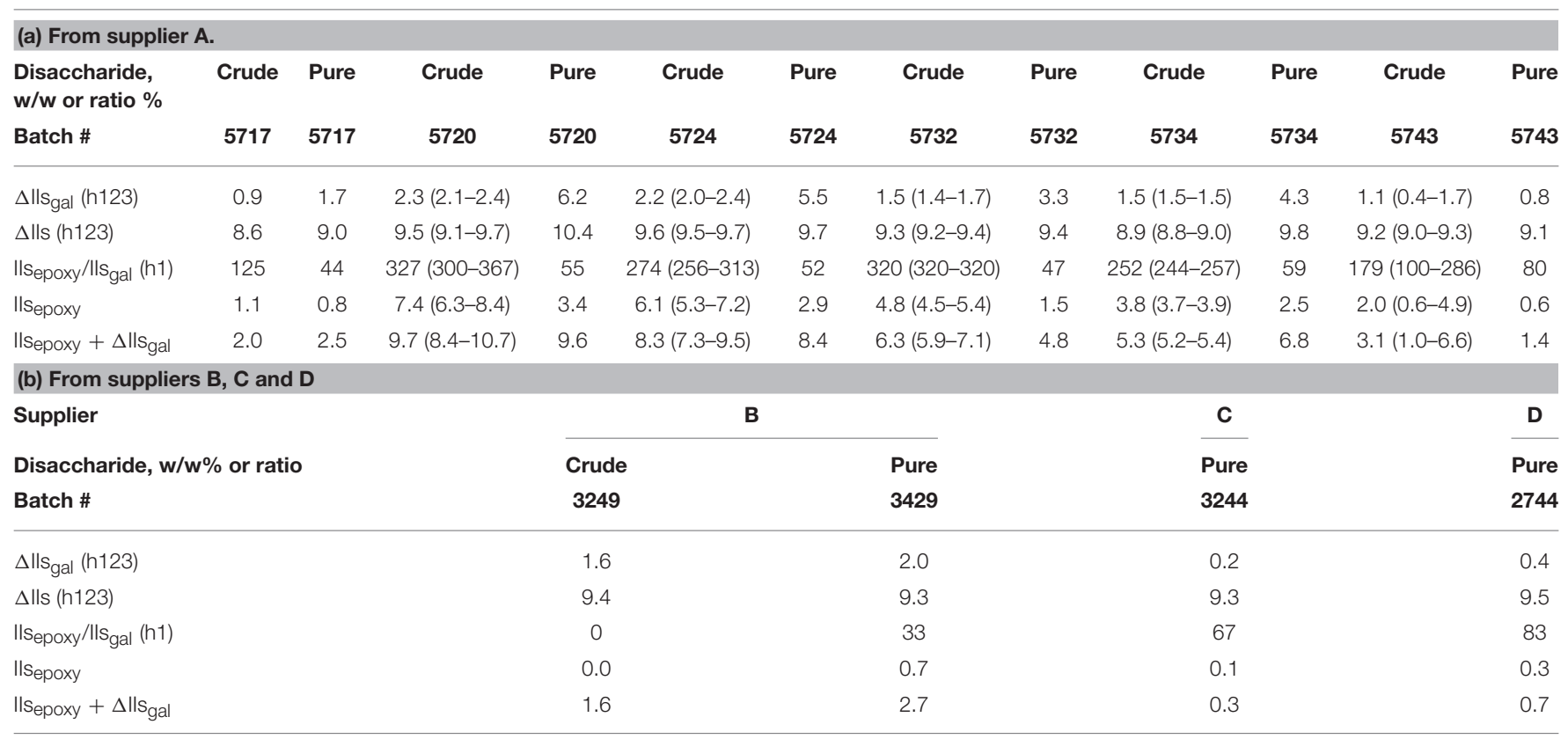

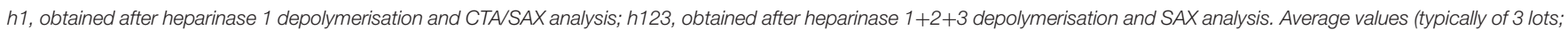
range is provided) for crude batches used to manufacture one pure heparin batch; batch number for this average crude batch is taken as same as corresponding pure batch.

step. The difficulty of the identification of IIs $s_{\text {epoxy }}$ in crude heparin samples, as previously discussed, explains why the degradation may remain "hidden" in crude heparin.

\section{Characterization of Desulfation Degradation Pathways in Heparin Samples}

The maximum $\Delta$ IIs content observed in pure heparin was 10.4\% (batch 5720; Table 1). This batch was derived from crude heparin batches with an initial content of 9.5\% (Table 2A). The increase of iduronic acid (IIs, k3 pathway) was therefore limited, and the selectivity of the epoxide ring opening was mostly toward the formation of galacturonic acid (IIs gal, k2) (Figure 1). In pure heparin batch 5720 (Tables 1, 2A), IIs epoxy $_{\text {-IIs }}$ gal reached $9.6 \%$ and $\mathrm{IVs}_{\text {gal }} 0.8 \%$, indicating that $>10 \%$ of the disaccharide units had been desulfated. This corresponds to the desulfation of $\sim 3$ disaccharide units per heparin chain containing an average 25 disaccharide units. There was no significant IIs $_{\text {epoxy }}$ in crude heparin from supplier B (Table 2B), whereas II $s_{\text {gal }}$ was observed at a concentration of $1-2 \%$, suggesting that the kinetics k1, k2, and k3 (Figure 1) greatly depend on the environment, i.e., the precise process conditions used when preparing crude heparin. Crude batches from suppliers $C$ and D (results not shown) had (IIs $s_{\text {epoxy }}+\mathrm{IIs}_{\text {gal }}$ ) content lower than $1.0 \%$, or very limited desulfation, further highlighting the differences in desulfation between different crude heparin suppliers.

IIs and IIs gal contents were correlated using data from supplier A samples in Table 1. The data confirmed that the preferred degradation pathway was toward IIs gal with $\mathrm{k} 2=6 \times \mathrm{k} 3,6$ being the reciprocal of the slope (0.17) (Figure 4A). As expected, and due to the transformation of the former into the latter, Is negatively-correlated with IIs gal (Figure 4B). Table 1 shows that the respective amounts of IIs $s_{\text {gal }}$ and IVs $s_{\text {gal }}$ (respectively 2.9 and $0.6 \%$ on average; $\frac{I I s_{\text {gal }}}{I V s_{\text {gal }}}$ ratio of 5) were not proportional to the content of their parent compounds, namely Is and IIIs (60.2 and $5.3 \%$ on average; $\frac{I s}{I I I s}$ ratio of 12$)$. Indeed, IV $_{\text {gal }}$ content observed was approximately twice what would have been expected from the respective contents of Is and IIIs. As a consequence, IV $s_{\text {gal }}$ should be monitored when performing a precise evaluation of 2-O desulfation.

\section{Characterization of Relationship Between \\ Desulfation, Anticoagulation, and Molecular Weight Measures in Pure Heparin Batches}

The relationship between disaccharide analysis data, activities (including anti-coag, anti-FXa, and anti-FIIa activities), and MW were evaluated for pure heparin batches from supplier A. Results obtained are presented in Table 1. Compliance with the Ph. Eur. monograph specifications is also listed (2).

Anti-FIIa activity appeared to be the most sensitive assessment tool for differentiating between varying levels of desulfation. The activity $(\mathrm{U} / \mathrm{mg})$ ranking was generally anti-FIIa $\leq$ antiFXa $\leq$ anti-coag. The anticoagulation data for the most desulfated batch (5720) suggested that such a batch could have passed the former Ph. Eur. anti-coag specification of $180 \mathrm{U} / \mathrm{mg}$, yet having only $150 \mathrm{U} / \mathrm{mg}$ anti-FIIa activity. The OSCS crisis (22) illustrated the value of specifying individual activity requirements, more specifically anti-FIIa activity which is now required by the USP and Ph. Eur. Pharmacopeias. Anti-FIIa activity negatively correlated with IIs $_{\text {gal }}$ levels, which could be explained by some structure-activity relationship (Figure 4C).

MW of the heparin batches ranged from 13,000 to 15,300 Da. Lower MW tended to be associated with lower anti-FIIa 


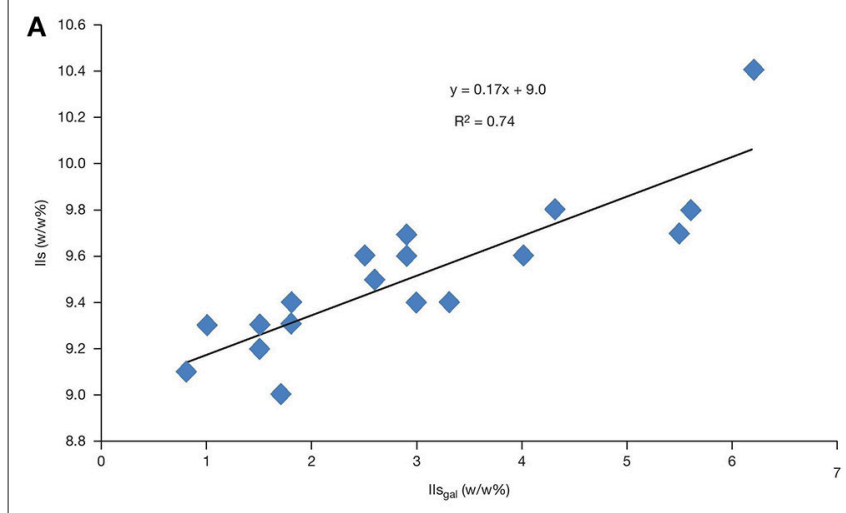

B

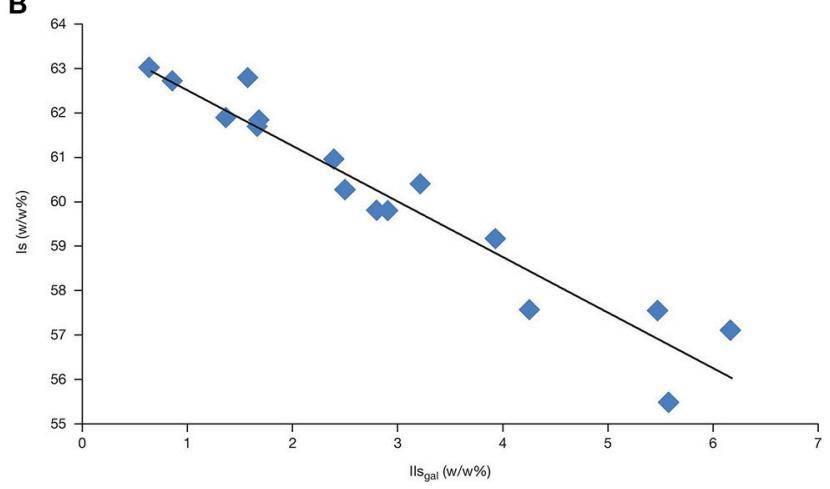

C

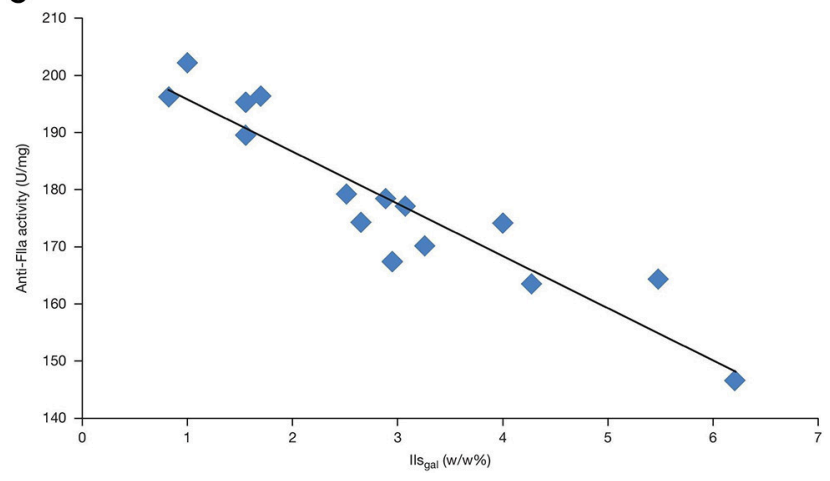

FIGURE 4 | Analyses of pure heparin from supplier A showing the correlations

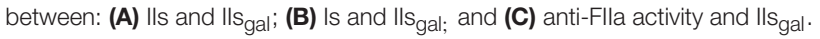

activity and vice versa; indeed, all batches with MW lower than $14,000 \mathrm{Da}$ had anti-FIIa activity lower than $180 \mathrm{U} / \mathrm{mg}$ and all batches with MW higher than 15,000 Da were associated with anti-FIIa activity higher than $195 \mathrm{U} / \mathrm{mg}$ (Table 1). This indicated that some depolymerization occurred concomitantly with desulfation, which could explain the reduction in antiFIIa activity. Hence, this is consistent with the requirement of chain lengths of at least 5,400 Da for triggering anti-FIIa activity. As a reminder, these absolute MW should not be directly compared with what could be obtained when using the current USP standard (not available at the time of this study).

Pure heparin batches obtained from crude batches of supplier A were expected to pass the 180 anti-FIIa U/mg USP (and Ph.
Eur.) specification with $\sim 4 \%$ desulfated disaccharides including $\sim 2.5 \%$ (Figure $4 \mathrm{C}$ ) $\mathrm{IIs}_{\text {gal }}, \sim 1.3 \%$ (Table 2) IIs $_{\text {epoxy, }}$, and $\sim 0.5 \%$ $\mathrm{IVs}_{\text {gal }}$ (Table 1) corresponding to an average of $\sim 1$ desulfated disaccharide per heparin chain. With another heparin supplier (pure heparin; data not presented), we could also link a rather low, although compliant anti-FIIa activity $(\geq 180 \mathrm{U} / \mathrm{mg})$ to the pattern of desulfation, up to a value of $\sim 5 \%$. Importantly our data indicate that, although all pure heparin batches show some evidence of desulfation, it is possible to obtain batches with very limited degradation indicating that these side reactions can be limited by appropriate process control.

\section{CONCLUSION}

Crude heparin is a very complex mixture of variable purity with limited relevant release controls. With usual controls, poor intrinsic quality of the heparin may only be revealed after processing into pure heparin. This investigation demonstrated, through a relevant set of quality parameters, that desulfation reactions resulting from alkaline environment occur at varying magnitude during process conditions typically encountered during industrial purification of heparin out of mucosa. We further reported two chromatographic techniques that, applied after two different conditions of heparinase depolymerization, can be used to assess the entire extent of desulfation at the crude and pure heparin stages. We showed that IIs gal and IIs $_{\text {epoxy }}$ are the main non-endogenous disaccharides formed, the latter being potentially especially present in crude heparin due to its partial hydrolysis during the purification process, where it is particularly challenging to analyze. Although process degradation impurities observed during these studies are present at varying levels in all marketed pure heparin sources due to related process conditions, their control is not directly addressed within the different monographs; this is also the case for the other structural process impurities. Indeed, we showed that a heparin with $4 \%$ desulfation, or 1 modified disaccharide per chain, could pass the current antiFIIa activity specification of the USP and Ph. Eur. monographs. To our knowledge, specific control of the process alteration of the heparin macromolecular backbone is not currently under consideration for future monograph evolution. Although disaccharide analysis is not systematically performed on each crude and pure heparin batch, our QC laboratory performs this analysis when qualifying a new supplier and when deemed justified on an at-risk basis (investigation, supplier control).

\section{AUTHOR CONTRIBUTIONS}

PA wrote the manuscript. CM performed the analyses. PM performed CTA-SAX analyses, contributed to and reviewed the manuscript. CV contributed to and reviewed the manuscript. All authors approved the submitted version.

\section{ACKNOWLEDGMENTS}

This study was funded by Sanofi. 


\section{REFERENCES}

1. USP Heparin Sodium Monograph. [Accessed June 2, 2018].

2. Ph. Eur. Heparin Sodium Monograph. [Accessed June 2, 2018].

3. Martinez C, Savadogo A, Agut C, Anger P. Reproducibility of the anti-Factor $\mathrm{Xa}$ and anti-Factor IIa assays applied to enoxaparin solution. J Pharm Biomed Anal. (2013) 81-82:138-45. doi: 10.1016/j.jpba.2013.03.021

4. Mourier P, Anger P, Martinez C, Herman F, Viskov C. Quantitative compositional analysis of heparin using exhaustive heparinase digestion and strong anion exchange chromatography. Anal Chem Res. (2015) 3:46-53. doi: 10.1016/j.ancr.2014. 12.001

5. Mourier P, Guichard O, Herman F, Viskov C. Heparin sodium compliance to the new proposed USP monograph: elucidation of a minor structural modification responsible for a process dependent $2.10 \mathrm{ppm}$ NMR signal. J Pharm Biomed Anal. (2011) 54:337-44. doi: 10.1016/j.jpba.2010. 09.011

6. Mourier P, Guichard O, Herman F, Viskov C. Heparin sodium compliance to the new proposed USP monograph: elucidation of a minor structural modification responsible for a process dependent $2.18 \mathrm{ppm}$ NMR signal. $J$ Pharm Biomed Anal. (2012) 67-68:169-74. doi: 10.1016/j.jpba.2012.04.015

7. Beccati D, Roy S, Lech M, Ozug J, Schaeck J, Gunay NS, et al. Identification of a novel structure in heparin generated by sequential oxidative-reductive treatment. Anal Chem. (2012) 84:5091-6. doi: 10.1021/ac3007494

8. USP <207> Test for 1,6 Anhydro Derivative for Enoxaparin Sodium. [Accessed June 2, 2018].

9. Ph. Eur. Enoxaparin Sodium Monograph. [Accessed June 2, 2018].

10. Mourier PA, Viskov C. Chromatographic analysis and sequencing approach of heparin oligosaccharides using cetyltrimethylammonium dynamically coated stationary phases. Anal Biochem. (2004) 332:299-313. doi: $10.1016 /$ j.ab.2004.06.020

11. USP $<208>$ Anti-Factor Xa and Anti-Factor IIa Assays for Unfractionated and Low Molecular Weight Heparins. [Accessed June 2, 2018].

12. Ph. Eur. §2.7.5. Assay of Heparin. [Accessed June 2, 2018].

13. Ph. Eur. \$5.3 Statistical Analysis. [Accessed June 2, 2018].

14. Toida TE, Vlahov IR, Smith AE, Hileman RE, Linhardt RJ. C-2 Epimerization of $\mathrm{N}$-acetylglucosamine in an oligosaccharides derived from heparan sulfate. J Carbohydr Chem. (1996) 15:351-60. doi: 10.1080/073283096080 05658
15. Yamada S, Watanabe M, Sugahara K. Conversion of N-sulfated glucosamine to $\mathrm{N}$-sulfated mannosamine in an unsaturated heparin disaccharide by non-enzymatic, base-catalyzed C-2 epimerization during enzymatic oligosaccharide preparation. Carbohydr Res. (1998) 309:261-8. doi: 10.1016/S0008-6215(98)00144-X

16. Rej R, Jaseja M, Perlin AS. Importance for blood anticoagulant activity of a 2 sulfate group on L-iduronic acid residues in heparin. Thromb Haemost. (1989) 61:540.

17. Desai UR, Wang HM, Kelly TR, Linhardt RJ. Structure elucidation of a novel acidic tetrasaccharide and hexasaccharide derived from a chemically modified heparin. Carbohydr Res. (1993) 241:249-59. doi: 10.1016/0008-6215(93)80112-R

18. Yamada S, Murakami T, Tsuda H, Yoshida K, Sugahara K. Isolation of the porcine heparin tetrasaccharideswith glucuronate 2-O-sulfate. J Biol Chem. (1995) 270:8696-705.

19. Mauri L, Boccardi G, Torri G, Karfunkle M, Macchi E, Muzi L, et al Qualification of HSQC methods for quantitative composition of heparin and low molecular weight heparins, J Pharm Biomed Anal. (2017) 136:92-105. doi: 10.1016/j.jpba.2016.12.031

20. Mauri L, Marinozzi M, Mazzini G., Kolinski R, Karfunkle M, Keire D, et al. Combining NMR spectroscopy and chemometrics to monitor structural features of crude heparin, molecules. (2017) 22:E1146. doi: 10.3390/molecules 22071146

21. Van Boeckel CAA, Petitou M. The unique antithrombin III binding domain of heparin: a lead to new synthetic antithrombotics. Angew Chem. (1993) 32:1671-818. doi: 10.1002/anie.199316713

22. Liu $\mathrm{H}$, Zhang $\mathrm{Z}$, Linhardt RJ. Lessons learned from the contamination of heparin. Nat Prod Rep. (2009) 26:313-21. doi: 10.1039/b819896a

Conflict of Interest Statement: PA, CM, and PM were employees of Sanofi, Paris, France. CV is now an employee of Aspen, Notre Dame de Bondeville, France.

Copyright (๑) 2018 Anger, Martinez, Mourier and Viskov. This is an open-access article distributed under the terms of the Creative Commons Attribution License (CC $B Y)$. The use, distribution or reproduction in other forums is permitted, provided the original author(s) and the copyright owner(s) are credited and that the original publication in this journal is cited, in accordance with accepted academic practice. No use, distribution or reproduction is permitted which does not comply with these terms. 


\section{APPENDIX}

\section{ABBREVIATIONS}

AT

BSA

Coag

CTA

EMA

FDA

Flla

FXa

GAGs

GPC

HPLC

LMWH

MW

NMR

OOS

OSCS

Ph. Eur.

SAX

SEC

UFH

USP

UV

$\mathrm{v} / \mathrm{v}$

W/w

Abb

$U$

IdoA

GlcA

$\triangle U$

$\Delta \mathrm{GlcA}$

GlcN

NS

NAC

2S

65

GalA

Epoxy

Structural symbols

IVs gal $_{\text {ga }}$

Ils gal

Ilsepoxy

Ils glu

IIIs $s_{\text {id }}$

IVs glu

$\| a_{i d}$

Illaid

$\mathrm{la}_{\mathrm{id}}$

Isid
Antithrombin

Bovine serum albumin

Coagulant

Cetyltrimethylammonium

European Medicines Agency

Food and Drug Administration

Factor lla

Factor Xa

Glycosaminoglycans

Gel permeation chromatography

High-performance liquid chromatography

Low-molecular-weight heparin

Mass average molecular weight

Nuclear Magnetic Resonance spectroscopy

Out of specification

Oversulfated chondroitin sulfate

European Pharmacopoeia

Strong anion exchange

Size exclusion chromatography

Unfractionated heparin

United States Pharmacopeia

Ultraviolet

Volume/volume

Weight/weight
The iduronic (id) or glucuronic (glu) structure of uronic acids is indicated for oligosaccharides, e.g., $\Delta \mathrm{Is}_{\mathrm{I}} \mathrm{III}_{\mathrm{id}}$

Main disaccharides are in the following table. Underlined disaccharides have a 3-O sulfated glucosamine, e.g., IIs glu (GlcAGlcNS,3S,6S).

$\Delta \mathrm{IVa}=\Delta \mathrm{U}-\mathrm{GICNAC}$

$\Delta \mathrm{IVs}$ gal $=\Delta \mathrm{GalA}-\mathrm{Gl} \mathrm{cNS}$

$\Delta \mathrm{IVs}=\Delta \mathrm{U}-\mathrm{Gl} \mathrm{CNS}$

$\Delta \mathrm{lla}=\Delta \mathrm{U}-\mathrm{GlcNAc}, 6 \mathrm{~S}$

$\Delta \mathrm{lll} \mathrm{a}=\Delta \mathrm{U} 2 \mathrm{~S}-\mathrm{Gl} \mathrm{cNAc}$

$\Delta \| \mathrm{s}_{\mathrm{gal}}=\Delta \mathrm{GalA}-\mathrm{GlcNS}, 6 \mathrm{~S}$

$\Delta \mathrm{lls}=\Delta \mathrm{U}-\mathrm{GlcNS}, 6 \mathrm{~S}$

$\Delta \mathrm{lll}=\Delta \mathrm{U} 2 \mathrm{~S}-\mathrm{GlcNS}$

$\Delta \mathrm{la}=\Delta \mathrm{U} 2 \mathrm{~S}-\mathrm{GlcNAc}, 6 \mathrm{~S}$

$\Delta \underline{\| l \mathrm{~S}}=\Delta \mathrm{U}-\mathrm{Gl} \mathrm{cNS}, 3 \mathrm{~S}, 6 \mathrm{~S}$

$\Delta \mathrm{ls}=\Delta \mathrm{U} 2 \mathrm{~S}-\mathrm{Gl} \mathrm{CNS}, 6 \mathrm{~S}$

$\Delta \underline{\mathrm{s}}=\Delta \mathrm{U} 2 \mathrm{~S}-\mathrm{Gl} \mathrm{cNS}, 3 \mathrm{~S}, 6 \mathrm{~S}$
Uronic acid

L-iduronic acid

D-glucuronic acid

4,5-unsaturated uronic acid, eg $\Delta$ GlcA

4,5-unsaturated glucuronic acid

D-glucosamine

N-sulfate

$\mathrm{N}$-acetyl

2-O-sulfate

6-O-sulfate

D-galacturonic acid

Epoxised iduronic acid

GalA-GIcNS

GalA-GlcNS,6S

GulA2,3epo -GlcNS,6S

GlcA-GlcNS,6S

IdoA-2S-GIcNS

GlcA-GIcNS

IdoA-GIcNAc,6S

IdoA-2S-GIcNAc

IdoA-2S-GIcNAc,6S

IdoA-2S-GIcNS,6S 